\title{
Comparison of meditation techniques for anxiety reduction in adults: An empirical study in Mexico
}

\author{
Carlos Ramón Galindo-López, Luis A. Castro, Cynthia B. Pérez, Jessica \\ Beltrán
}

Published: 30 November 2021

\begin{abstract}
The number of people who experience anxiety disorders around the world has increased in the last few years. Studies report that meditation can reduce stress or anxiety. There are several videos on online platforms such as YouTube stating that they can reduce anxiety or stress. In this work, we present the results of a comparison between mediation at home following videos that can be found on YouTube and other methods for reducing anxiety such as their individuals' preferred methods. Our results suggest that both methods are comparable, and that a few minutes of their own preferred methods for reducing anxiety can be as effective as a YouTube video. The methods and findings of this work can be of interest for testing applications and other techniques that may exist for reducing anxiety in users.
\end{abstract}

\section{Keywords:}

Anxiety; Experimental design; Guided meditation.

\section{Introducción}

Se estima que la población mundial con trastornos de ansiedad en 2015 fue del 3.6\%, equivalente a 264 millones de personas. Este total refleja un incremento del $14.9 \%$ desde 2005 [10]. La ansiedad es una respuesta del estrés, pero si persiste por más de seis meses es posible que padezca un trastorno de ansiedad. El tratamiento para la ansiedad se divide en farmacológicos (P.Ej. medicación) y no farmacológicos (P.Ej. psicoterapia). Los medicamentos para prevenir los síntomas de la ansiedad incluyen antidepresivos y sedantes. Sin embargo, estudios realizados indican que el uso de meditación ofrece una reducción de la ansiedad, mejora el rendimiento académico $[1,2,4]$ o reducción del estrés [7, 11].

Diversas plataformas basadas en aplicativos en las tiendas de App Store o el Google Play proveen videos o técnicas para

Galindo-López, Carlos Ramón., Castro, Luis A., Pérez, Cynthia B.

Instituto Tecnológico de Sonora (ITSON)

Ciudad Obregón, México

carlos.galindo147341@potros.itson.edu.mx, luis.castro@acm.org, cynthia.perez@itson.edu.mx

Beltrán, Jessica.

Consejo Nacional de Ciencia y Tecnología - Instituto Politécnico Nacional

Tijuana, México

jbeltran@citedi.mx reducción de ansiedad en usuarios. De igual manera, muchos de este tipo de videos se pueden encontrar en plataformas comerciales como YouTube. Sin embargo, no es claro si muchos de estos videos son efectivos en la reducción de ansiedad en la audiencia.

En este trabajo se presenta un experimento para el estudio de los efectos de la meditación guiada en los niveles de ansiedad de adultos por medio de un video de la plataforma de YouTube, cuando se comparan con los métodos personales como respiración profunda o actividad física, los cuales a menudo son utilizados para la reducción de ansiedad de los participantes.

\section{Trabajo relacionado}

En la literatura típicamente se conocen dos tipos de ansiedad: ansiedad estado y ansiedad rasgo. La ansiedad estado es cuando la persona tiene sentimientos de miedo, nerviosismo e incomodidad y la respuesta fisiológica asociada se da en forma de incremento de la activación del sistema nervioso autónomo mediante un estímulo o situación percibido como potencialmente peligrosa. Por otro lado, la ansiedad rasgo se refiere cuando la persona tiene esos mismos sentimientos, pero no por un estímulo percibido como peligroso en el entorno, sino porque la persona tiene una predisposición de personalidad a sentirse ansiosa. Se han realizado diversos trabajos que están relacionados con el estudio de la meditación para reducir ansiedad en adultos.

Por ejemplo, se estudió la reducción de la ansiedad siguiendo ejercicio y meditación [1]. En la investigación participaron 75 hombres adultos de edades entre 22 y 71 años, 25 asignados a grupos de control, ejercicio o meditación. Los participantes del grupo de control descansaron en una silla lazyboy durante 20 minutos. Los resultados sugieren que la actividad física aguda, meditación y una sesión de descanso tranquila son formas efectivas de reducir la ansiedad estado. De igual manera, también se ha estudiado la meditación de atención plena, ya que es una técnica psicológica basada en ejercicios para mantener la atención focalizada en el tiempo presente. En el estudio presentado por Beauchemin et al. utilizan esta técnica para disminuir la ansiedad, promover las habilidades sociales y mejorar el rendimiento académico entre los adolescentes con capacidades diferentes [2]. Este estudio con 34 adolescentes diagnosticados con dificultades de aprendizaje de edades desde 13 hasta 18 años y de una duración de 5 semanas; se aplicó la meditación plena a los participantes logrando reducir la ansiedad estado y rasgo, mejora de las habilidades sociales y mejora del rendimiento académico para los participantes que completaron el programa. 
También, la meditación se ha usado para aliviar de manera natural el estrés como herramienta para reducir la ansiedad y aumentar la autorrealización [4]. En el estudio se utilizó la meditación a un grupo de 31 participantes $(25$ hombres y 6 mujeres); después de 4 semanas de práctica regular se obtuvo una reducción significativa de la ansiedad rasgo medida por el cuestionario STAI el cual se aplica a los participantes con el objetivo de conocer el nivel de ansiedad estado y rasgo [8].

Además de la meditación, diferentes métodos se han utilizado para la reducción de ansiedad, por ejemplo se han estudiado los efectos de una sesión de ejercicio [11]. Los resultados indican que una sola sesión de yoga puede tener una eficacia pequeña a moderada para reducir ansiedad.

Además de meditación y actividad física, también se ha estudiado el uso de aplicaciones móviles. Por ejemplo, se han estudiado los efectos en el bienestar mental y el estrés percibido al utilizar la meditación plena por medio de la aplicación HeadSpace [12]. Este estudio, con 64 estudiantes (55 mujeres y 9 hombres, un $79.7 \%$ con edades entre $18-25$ años y $20.3 \%$ con edades mayores de 25 años), muestra que la meditación plena al aplicarse por al menos 10 minutos al día durante 4 semanas se asocia con una mayor atención y bienestar mental, así como una disminución del estrés. Sin embargo, es importante señalar que no todas las aplicaciones comerciales necesariamente han sido diseñadas por profesionales [9]. Pocos trabajos se han encontrado con el uso de videos como técnicas para reducción de ansiedad. Uno de los pocos artículos que estudian los videos como manera de meditar es el trabajo descrito en [3], en el que se estudian los comentarios publicados en 89 videos y 1,144 comentarios de los que se desprenden categorías como comentarios acerca del video o reportes de experiencias subjetivas derivadas del video. Sin embargo, aunque se ha estudiado que la meditación puede tener efectos positivos en la reducción de ansiedad o estrés, aun no es claro si videos realizados con propósitos de meditación logran reducir la ansiedad en usuarios. Más aún, no es claro si los videos que se encuentran en YouTube pueden ser comparables a otras técnicas de reducción de ansiedad utilizadas por los propios usuarios.

\section{Métodos}

En esta sección, se presentan los métodos utilizados en este trabajo con el objetivo de estudiar los efectos de la meditación guiada en los niveles de ansiedad de adultos por medio de un video de la plataforma de YouTube, cuando se comparan con los métodos personales de reducción de ansiedad de los participantes.

El diseño del experimento fue between-subjects, por lo que hay dos grupos de usuarios sujetos a distintas condiciones que a continuación se presentan.

\subsection{Variables Independientes}

Las variables independientes bajo estudio Tipo de Meditación (2 niveles):

- Meditación guiada: Técnica de meditación guiada por un video instruccional disponible en plataforma comercial.

- Métodos de reducción de ansiedad personales: Técnica que utiliza la persona para meditación. Es decir, reducir ansiedad por sí mismo, utilizando su método de preferencia (no farmacológicas).

\subsection{Variables dependientes}

- Ansiedad estado: . La ansiedad-estado suele ser cuando la persona tiene sentimientos de miedo, nerviosismo e incomodidad siendo una respuesta adaptativa que acaba desapareciendo tras haberse dejado atrás la amenaza [5].

- Ansiedad rasgo: La ansiedad-rasgo es un concepto que, en términos de síntomas, no se diferencia demasiado de su homóloga la ansiedad-estado, solo que el origen de esta reacción emocional es distinto. En este caso, la persona suele estar en tensión constantemente debido a su personalidad, es su día a día [5].

\subsection{Hipótesis}

- H1: Los niveles de ansiedad estado entre el grupo de tratamiento y el grupo de control serán significativamente diferentes.

- H2: Los niveles de ansiedad rasgo entre el grupo de tratamiento y el grupo de control serán significativamente diferentes.

\subsection{Participantes}

Para la realización del experimento se reclutaron 16 personas $(8$ hombres y 8 mujeres) en el noroeste de México con edades mayores o iguales a 18 años y menores de 65 , mediante muestreo por conveniencia. Los participantes se dividieron de manera aleatoria en el grupo experimental $(\mathrm{N}=8)$ y el grupo de control $(\mathrm{N}=8)$. Es decir, 8 participantes utilizaron el video de meditación guiada, y los 8 restantes utilizaron una técnica de reducción de ansiedad de su preferencia.

\subsection{Instrumentos}

En esta sección se describen los instrumentos utilizados durante el experimento.

- Prueba Stroop: Se utiliza al inicio de cada experimento para inducir cierto nivel de ansiedad, y tratar de nivelar a todos los participantes. La prueba consiste en 3 etapas: 1) la primera etapa se le dan nombres de colores en tinta negra. Por ejemplo, "rojo" o "azul" y hay que decir la palabra por 45 segundos empezando de derecha a izquierda y de arriba abajo; 2) En la segunda etapa se muestran filas de varios colores y se tiene que decir el color de la fila por la misma duración durante el resto de la etapa; 3) En la última etapa se dan nombres de colores que se presentan en un color distinto al que corresponde la palabra escrita y hay que ignorar lo que dice la palabra y en cambio debe decir el color del texto.

- Video para meditación guiada: Se utiliza el video llamado "Relajación Guiada para la Ansiedad 10 Minutos de Calma" del canal llamado Psicología Online ${ }^{1}$. El video tiene una duración de 10 minutos y se encuentra disponible libremente.

- STAI:, El cuestionario STAI [8] está constituido por dos escalas, midiendo la ansiedad-estado y la ansiedad-rasgo, teniendo la ansiedad estado 18 ítems y la ansiedad rasgo 20 ítems, haciendo un total de 38. Los ítems se responden en escala Likert, de 0 a 3 . Algunos ítems son negativos y por lo tanto se utiliza un valor negativo. Las puntuaciones van desde -27 hasta 27 para la ansiedad estado y desde -18 hasta 42 . A mayor puntuación, mayor ansiedad.

\footnotetext{
${ }^{1}$ Enlace: https://www.youtube.com/watch?v=GCzvhUqJpsA
} 


\subsection{Procedimiento}

El experimento para ambos grupos consistió en una sesión en la plataforma y horario de conveniencia de los participantes donde se le explica la dinámica a realizar.

A ambos grupos se les solicitó realizar la prueba Stroop [6]. Luego, se les preguntó sobre su método preferido para reducir ansiedad, así como la frecuencia de uso. También se les preguntó sobre si tenían experiencia con la meditación guiada.

Enseguida contestaron el cuestionario STAI [8]. Al finalizar el cuestionario STAI los participantes intentaron reducir la ansiedad utilizando el método de la condición de su grupo durante 10 minutos (duración del video para el grupo de tratamiento). Al finalizar los 10 minutos se aplicó de nuevo el cuestionario STAI para conocer el nivel de ansiedad al finalizar la sesión.

\subsection{Análisis de los datos}

Los datos se analizaron con una prueba $\mathrm{t}$ de student para grupos independientes cuando se compararon entre técnicas de meditación, y se utilizó la prueba t de student emparejadas cuando se hicieron comparaciones pre y post test entre los mismos individuos.

\section{Resultados}

En esta sección se muestran los resultados por tipo de ansiedad.

\subsection{Ansiedad estado}

Se compararon los grupos para ver si existían diferencias previas a realizar cada una de las condiciones. El nivel de ansiedad estado fue más alto en la técnica meditación guiada (media $=-1.00$, $\mathrm{DE}=10.57$ ) que en la meditación personal (media $=-3.75$, $\mathrm{DE}=6.45)$. Sin embargo, una prueba $\mathrm{t}$ independientes mostró que no hay una diferencia significativa $(\mathrm{t}=-.628, \mathrm{df}=11.582, \mathrm{p}=.542$, dos colas), por lo que el nivel de ansiedad estado era comparable en ambos grupos antes de iniciar con el experimento.

Tabla 1. Ansiedad estado en pre y post-test

\begin{tabular}{|l|l|r|r|}
\hline Condición & & Pre & \multicolumn{1}{c|}{ Post } \\
\hline $\begin{array}{l}\text { Meditación } \\
\text { Guiada }\end{array}$ & Media & -1.00 & -8.63 \\
\cline { 2 - 4 } & $\mathrm{N}$ & 8 & 8 \\
\cline { 2 - 4 } & $\begin{array}{l}\text { Desv. } \\
\text { Est. }\end{array}$ & 10.57 & 8.67 \\
\hline Personal & Media & -3.75 & -7.63 \\
\cline { 2 - 4 } & $\mathrm{N}$ & 8 & 8 \\
\hline \multirow{5}{*}{ Total } & Desv. & 6.45 & 5.24 \\
& Est. & & \\
\hline & Media & -2.38 & -8.13 \\
\hline & $\mathrm{N}$ & 16 & 16 \\
\hline & Desv. & 8.58 & 6.94 \\
\hline
\end{tabular}

En la Tabla 1 se muestran los resultados descriptivos referentes a ansiedad estado. La media de los niveles de ansiedad estado para la técnica de meditación guiada fue de $-1.00(\mathrm{DE}=10.57)$ antes del experimento (pre-test) y $-8.63(\mathrm{DE}=8.67)$ para después del experimento (post-test). La diferencia de medias antes y después del experimento para meditación guiada fue de $7.63 \mathrm{y}$ el intervalo de confianza de $95 \%$ de la diferencia de medias esta entre 4.59 y 10.65. Una prueba $\mathrm{t}$ de muestras emparejadas mostró que la diferencia entre las condiciones fue significativa $(\mathrm{t}=5.953, \mathrm{df}=7$, $\mathrm{p}=.001$, dos colas)

La media de los niveles de ansiedad estado para la técnica personal fue de $-3.75(\mathrm{DE}=6.45)$ antes del experimento (pre-test) y -7.63 (DE=5.24) para después del experimento (post-test). La diferencia de medias antes y después del experimento para la técnica personal fue de 3.88 y el intervalo de confianza de $95 \%$ de la diferencia de medias esta entre -0.97 y 8.72 . Una prueba $t$ emparejada mostró que la diferencia entre las condiciones no fue significativa $(\mathrm{t}=-1.892, \mathrm{df}=7, \mathrm{p}=.100$, dos colas).

Siguiendo la Figura 1, se puede observar una disminución en el nivel de ansiedad estado de los participantes independientemente del tipo de técnica utilizada para meditación. Al comparar los totales, la media de los niveles de ansiedad estado fue de -2.38 (DE $=8.58$ ) antes del experimento (pre-test) para ambas condiciones y de $-8.13(\mathrm{DE}=6.94)$ para después de las condiciones (post-test). La diferencia de medias antes y después del experimento fue de $5.75 \mathrm{y}$ el intervalo de confianza de $95 \%$ de la diferencia de medias está entre 3.05 y 8.44. El tamaño del efecto es grande $(\mathrm{d}=0.74)$. Una prueba $t$ mostró que la diferencia entre las condiciones fue significativa $(\mathrm{t}=4.552, \mathrm{df}=15, \mathrm{p}<.001$, dos colas $)$.

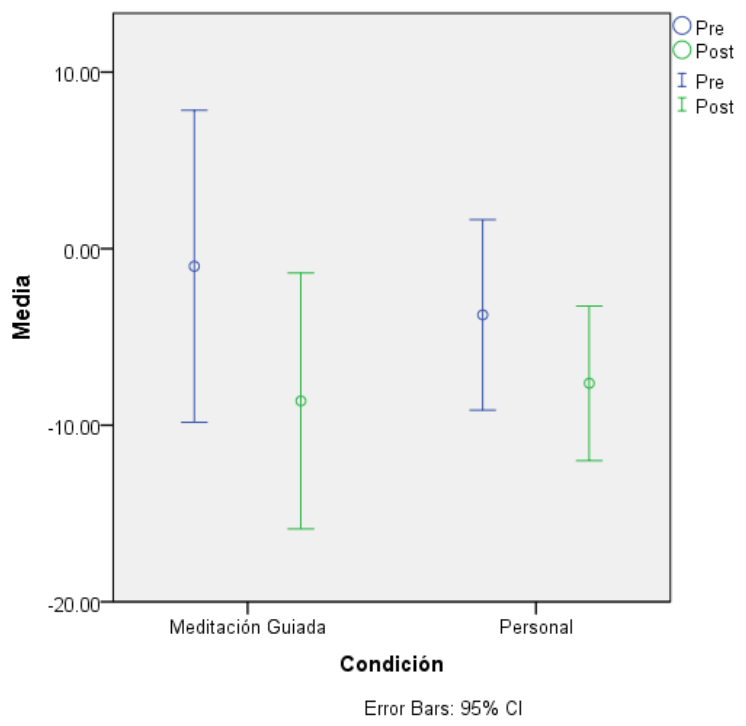

Figura 1. Ansiedad estado pre y post-test

Al comparar el nivel de ansiedad estado después del experimento es más alto en la técnica personal (media $=-7.63$ ) que en la técnica de meditación guiada (media $=-8.63$ ). Sin embargo, una prueba $t$ independiente mostró que dicha diferencia de medias no es significativa $(\mathrm{t}=.279, \mathrm{df}=14, \mathrm{p}=.784$, dos colas).

\subsection{Ansiedad rasgo}

Se compararon los grupos para ver si existían diferencias previas a realizar cada una de las condiciones. El nivel de ansiedad rasgo fue más alto en la técnica Personal (media $=8.63, \mathrm{DE}=13.84)$ que en la meditación guiada (media $=1.13, \mathrm{DE}=8.63$ ). Sin embargo, una prueba $\mathrm{t}$ independiente mostró que no hay una diferencia significativa $(\mathrm{t}=--1.301, \mathrm{df}=14, \mathrm{p}=.214$, dos colas $)$, por lo que el nivel de ansiedad rasgo era comparable entre ambos grupos antes de iniciar con el experimento.

Tabla 2. Ansiedad rasgo en pre y post-test

\begin{tabular}{|l|l|c|c|}
\hline Condición & Pre & Post \\
\hline & Media & 8.63 & 4.50 \\
\hline \multicolumn{3}{|c}{ aMPCihe }
\end{tabular}




\begin{tabular}{|l|l|r|r|}
\hline \multirow{2}{*}{$\begin{array}{l}\text { Meditación } \\
\text { Guiada }\end{array}$} & $\mathbf{N}$ & 8 & 8 \\
\cline { 2 - 4 } & $\begin{array}{l}\text { Desv. } \\
\text { Est. }\end{array}$ & 13.84 & 11.72 \\
\hline Personal & Media & 1.13 & -1.88 \\
\hline & N & 8 & 8 \\
\cline { 2 - 4 } & $\begin{array}{l}\text { Desv. } \\
\text { Est. }\end{array}$ & 8.63 & 7.99 \\
\hline \multirow{2}{*}{ Total } & Media & 4.88 & 1.31 \\
\hline & N & 16 & 16 \\
\hline & Desv. & 11.79 & 10.24 \\
\hline & Est. & & \\
\hline
\end{tabular}

En la Tabla 2 se muestran los resultados descriptivos referentes a ansiedad rasgo. La media de los niveles de ansiedad rasgo para la meditación guiada fue de $8.63(\mathrm{DE}=13.84)$ antes del experimento (pre-test) para ambas condiciones y de $4.50(\mathrm{DE}=11.72)$ para después de las condiciones (post-test). La diferencia de medias antes y después del experimento fue de $4.13 \mathrm{y}$ el intervalo de confianza de $95 \%$ de la diferencia de medias esta entre 0.02 y 8.23 . Una prueba t mostró que la diferencia entre las condiciones fue significativa $(\mathrm{t}=2.375, \mathrm{df}=7, \mathrm{p}=.049$, dos colas).

La media de los niveles de ansiedad rasgo para la técnica personal fue de $1.13(\mathrm{DE}=8.63)$ antes del experimento (pre-test) para ambas condiciones y de $-1.88(\mathrm{DE}=7.99)$ para después de las condiciones (post-test). La diferencia de medias antes y después del experimento fue de $3.00 \mathrm{y}$ el intervalo de confianza de $95 \%$ de la diferencia de medias está entre 1.16 y 4.84 . Una prueba t mostró que la diferencia entre las condiciones fue significativa $(t=8.850$, $\mathrm{df}=7, \mathrm{p}=.006$, dos colas).

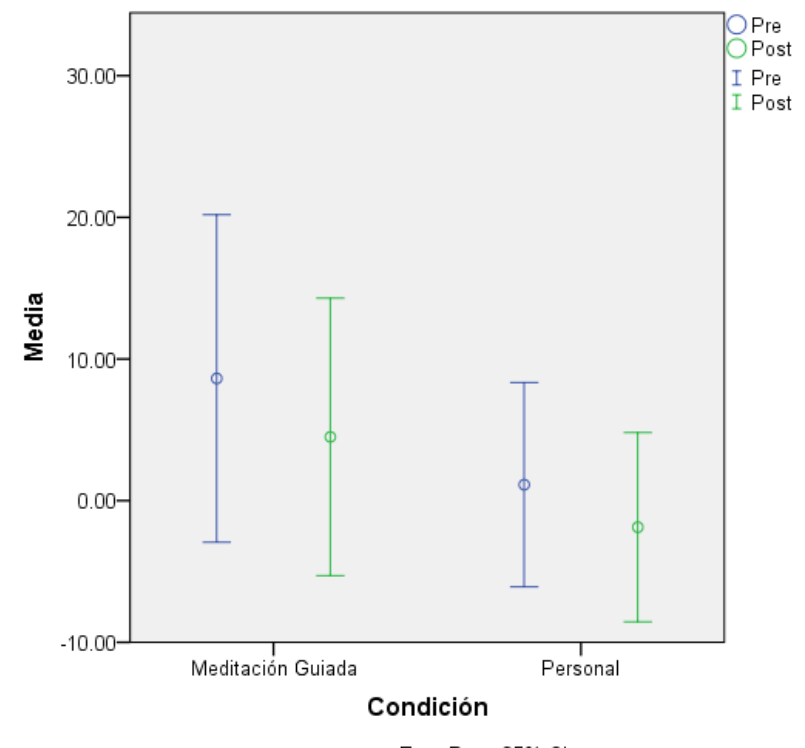

Error Bars: $95 \% \mathrm{Cl}$

Figura 2. Ansiedad rasgo pre y post-test

Siguiendo la Figura 2, se puede observar una disminución en el nivel de ansiedad rasgo de los participantes independientemente del tipo de técnica utilizada para meditación. Al comparar los totales, la media de los niveles de ansiedad rasgo fue de 4.88 (DE $=11.79$ ) antes del experimento (pre-test) para ambas condiciones y de $1.31(\mathrm{DE}=10.24)$ para después de las condiciones (post-test). La diferencia de medias antes y después del experimento fue de
3.56 y el intervalo de confianza de $95 \%$ de la diferencia de medias está entre 1.58 y 5.55. El tamaño del efecto es mediano $(\mathrm{d}=0.32)$. Una prueba $t$ mostró que la diferencia entre las condiciones fue significativa $(\mathrm{t}=3,827, \mathrm{df}=15, \mathrm{p}=.002$, dos colas $)$.

Al comparar el nivel de ansiedad rasgo después del experimento es más alto en la técnica de meditación guiada (media $=4.50$ ) que en la técnica personal (media $=-1.88$ ). Sin embargo, una prueba $t$ independiente mostró que dicha diferencia de medias no es significativa $(\mathrm{t}=-1.271, \mathrm{df}=14, \mathrm{p}=.224$, dos colas $)$.

\section{Discusión}

Los resultados de este experimento muestran que las técnicas de reducción de ansiedad de este estudio son comparables entre sí para nuestros participantes. Independientemente de la técnica utilizada, los participantes lograron reducir su nivel de ansiedad cuando se comparan sus mediciones pre y post-test. De igual forma, al comparar el desempeño de ambas técnicas directamente en su evaluación post-test, se puede observar que no hay diferencia significativa entre ellas, tanto para ansiedad estado como para ansiedad rasgo.

Sin embargo, al comparar los resultados que se obtuvieron, se puede ver que los resultados en ansiedad estado solamente fueron significativos para aquellos participantes que utilizaron la técnica de meditación guiada. Por otro lado, los participantes pudieron reducir significativamente sus niveles de ansiedad estado en ambas condiciones.

Este tipo de experimentos puede ser utilizado para medir la efectividad y desempeño de muchas aplicaciones comerciales que están orientadas a la disminución de ansiedad. Los resultados de este trabajo sugieren que técnicas de reducción de ansiedad de cada individuo pueden ser tan efectivas como técnicas de meditación guiada en algunos casos. Los resultados que se obtuvieron de nuestros participantes pueden tener varias aplicaciones en el área de interacción humano computadora. Por ejemplo, al haber observado un efecto en la ansiedad rasgo, debido a que es presumiblemente un aspecto temporal, se puede utilizar sistemas interactivos para proveer técnicas de meditación guiada, y a la vez estar monitorizando sus niveles de ansiedad. De igual forma, se pueden pensar en terapias asistidas por computadora para la reducción de los niveles de ansiedad. Es importante identificar las preferencias de los usuarios en cuanto a técnicas de reducción de ansiedad, ya que algunos pueden preferir técnicas como respiración profunda.

Además, es importante identificar futuras líneas de investigación que estén relacionadas con el diseño de tecnologías interactivas, las cuales pudieran ser más efectivas que las técnicas personales de reducción de ansiedad ya que podría estarse monitorizando mediante tecnologías vestibles.

\subsection{Limitaciones}

Este estudio tuvo varias limitaciones, las cuales describimos. Primero, la novedad o familiaridad con las técnicas de meditación elegida pudo haber afectado el desempeño de los participantes. Además, no se tuvo control sobre la técnica de reducción de ansiedad elegida por los participantes. El experimento se realizó en un solo día por lo que los resultados están modulados por los niveles de ansiedad en los que llega el participante al experimento, los cuales se pueden ver influidos por eventos externos que están fuera del control de los experimentadores. Finalmente, este estudio se pudo haber beneficiado de una muestra más grande. 


\section{Conclusiones}

En este trabajo, se presentaron los resultados de comparar la meditación en casa siguiendo un video que se puede encontrar en la plataforma de YouTube en comparación con los propios métodos de reducción de ansiedad que utilizan las propias personas. Se puede concluir que las técnicas para reducir ansiedad en casa tienen el potencial de ser tan efectivos como los videos que se encuentran en plataformas comerciales como YouTube.

Como trabajo futuro se planea explorar otros tipos de técnicas para reducir ansiedad, así como plantear tecnologías interactivas que permitan mejorar la experiencia de usuario al momento de estar realizando técnicas para reducción de ansiedad.

\section{Agradecimientos}

A los participantes del estudio. También, se agradece al Consejo Nacional de Ciencia y Tecnología (CONACYT) por la beca al primer autor. Este proyecto fue financiado parcialmente por el Instituto Tecnológico de Sonora (ITSON) por medio de PROFAPI.

\section{Referencias}

[1] Bahrke, M.S. and W.P. Morgan, Anxiety Reduction Following Exercise and Meditation. Cognitive Therapy and Research, 1978. 2(4): p. 323-333.

[2] Beauchemin, J., T.L. Hutchins, and F. Patterson, Mindfulness meditation may lessen anxiety, promote social skills, and improve academic performance among adolescents with learning disabilities. Complementary Health Practice Review, 2008. 13(1): p. 34-45.

[3] Buie, E. and M. Blythe. Meditations on YouTube. in Proc. of the 6th International Conference on Designing Pleasurable Products and Interfaces (DPPI 2013). 2013.

[4] Coppola, F. and D. Spector, Natural stress relief meditation as a tool for reducing anxiety and increasing self-actualization. Social Behavior and Personality, 2009. 37(3): p. 307-312.

[5] Elwood, L.S., K. Wolitzky-Taylor, and B.O. Olatunji, Measurement of anxious traits: A contemporary review and synthesis. Anxiety, Stress and Coping, 2012. 25(6): p. 647-666.

[6] Golden, C.J., Stroop, test de colores y palabras. 6th edition ed2020, Madrid, Spain: TEA Ediciones, S.A.

[7] Melville, G.W., et al., Fifteen minutes of chair-based yoga postures or guided meditation performed in the office can elicit a relaxation response. Evidence-Based Complementary and Alternative Medicine, 2012. 2012.

[8] Spielberger, C., R. Gorsuch, and R. Lushene, STAI, Manual for the State-Trait Anxiety Inventory (Self Evaluation Questionnaire). Adaptación española. ed1982, Madrid: TEA Ediciones S.A.

[9] Sucala, M., et al., Anxiety: There is an app for that. A systematic review of anxiety apps. Depression and anxiety, 2017. 34(6): p. 518-525.

[10] World Health Organization, Depression and Other Common Mental Disorders Global Health Estimates. 2017.

[11] Yin, J., L. Tang, and R.K. Dishman, The effects of a single session of mindful exercise on anxiety: A systematic review and meta-analysis. Mental Health and Physical Activity, 2021. 21.

[12] Zollars, I., T.I. Poirier, and J. Pailden, Effects of mindfulness meditation on mindfulness, mental wellbeing, and perceived stress. Currents in Pharmacy Teaching and Learning, 2019. 11(10): p. 1022-1028. 